\title{
Research of Modeling Technique for Waveform Distortion of Navigation Signal in Time Domain
}

\author{
Luo XianZhi \\ State Key Laboratory of Satellite Navigation System \\ and Equipment Technology \\ The $54^{\text {th }}$ Institute of China Electronic Technology \\ Corporation \\ Shijiazhuang, China \\ e-mail: 18632190780@163.com
}

Xie Jian

State Key Laboratory of Satellite Navigation System and Equipment Technology

The $54^{\text {th }}$ Institute of China Electronic Technology

Corporation

Shijiazhuang, China

e-mail: xiejian8901@126.com

\author{
Chao Lei \\ State Key Laboratory of Satellite Navigation System \\ and Equipment Technology \\ The $54^{\text {th }}$ Institute of China Electronic Technology \\ Corporation \\ Shijiazhuang, China \\ e-mail: chaolei001@163.com \\ Liu Liang \\ State Key Laboratory of Satellite Navigation System \\ and Equipment Technology \\ The $54^{\text {th }}$ Institute of China Electronic Technology \\ Corporation \\ Shijiazhuang, China \\ e-mail: liuliang1989@sohu.com
}

\begin{abstract}
This paper introduces a chip waveform distortion model of satellite navigation signal in time domain that is adopted by ICAO, and then proposes a FPGA implementation method with which three categories of chip waveform distortion of navigation signal in time domain are modeled. A comparison is made between the MATLAB simulation result and the FPGA implementation result of the chip waveform distortion after they are given. The test results show that their spectrums are in agreement in time domain waveform and frequency domain.
\end{abstract}

Keywords-satellite navigation; signal distortion; waveform distortion in time domain; ICAO threat model

\section{INTRODUCTION}

As the most critical factor in a satellite navigation system, the quality of satellite navigation signal is directly related to the receiving, demodulation, pseudo-random code ranging, carrier phase measurement and positioning solution performance of navigation signal for user, eventually affecting user's experience in positioning, navigation and time service. In case of performance degradation in navigation, positioning or time service, it can be ultimately attributed to the abnormality of navigation signal, which is essentially reflected in the abnormality of navigation signal base band in time domain. The causes to abnormality of navigation signal can be categorized into three types: signal transmitting abnormality at satellite payload, signal distortion caused by environment and effect from receiving system. The last two causes can be generalized to environment effect to navigation signal. To complete classification as well as mechanism and behavior analysis of navigation signal abnormities, it is required to propose various signal threat models on the premise of eliminating the effect of testing environment, thus to locate system failures. The oversea research work in the field of classification as well as mechanism and behavior analysis of navigation signal abnormities has lasted for about 10 years and put forward many effective navigation signal threat models. The mapping relation between signal abnormality and load faulty of navigation satellite has been established as well. The ICAO also requires parametric the waveform abnormality of GPS and GLONASS in time domain in the Convention on International Civil Aviation. Comparing with the research efforts abroad, it is still a blank in this field in China; therefore, it is necessary to carry out research of the waveform abnormality of navigation signal in time domain.

\section{TIME-DOMAIN WAVEFORM DiSTORTION MODEL OF SATELLITE NAVIGATION SIGNAL}

The nonlinearity of satellite signal transmission link will cause waveform distortion of signal chip, typically for example, the signal abnormality event of \#19 satellite happened in $1993^{[1]}$. To explain the signal abnormity of \#19 satellite, researchers proposed three threat models of navigation signal early or late: Simple Threat Model [2], MEWF[3](Most Evil Waveform) Threat Model and 2OS(2nd-Order Step) Threat Model. The Simple Threat Model is defined by superposing a signal on a standard navigation signal, which can't fully reflect all the characteristics of an abnormal signal. The MEWF Threat Model is defined as the signal waveform that the maximum differential pseudo-range error will come up for a specific user receiver. Mitelman ${ }^{[3]}{ }^{[4]}$ and Enge ${ }^{[5]}$ et.al. derived the mathematical expression of MEWF Threat 
Model particularly; However, this model is non-causal, difficult to realize. Therefore, neither the ICAO nor the International Commission on Air Navigation adopts this model. By contrast, 2OS Threat Model can reflect all the cases of satellite circuit failures more inclusively. The general mathematical model of payload is as shown in Fig .1.

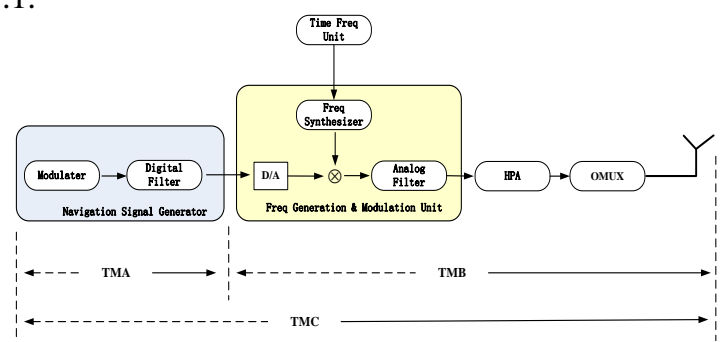

Figure 1. Payload model

In the model, the navigation signal generation unit produces navigation baseband signals, with the transmitted bandwidth equal to the bandwidth of the digital filter. The frequency generation and modulation unit converts the digital signals into analog signals and modulates the signals to the carrier frequency by means of several orders of up-conversion. The HPA(usually TWTA or SSPA) amplifies the signal power to saturation level typically. The OMUX Output Multiplexer combines the navigation signals at adjacent frequency points and couples it to one the same antenna for output.

In case of abnormality with any part of the payload, signal distortion might occur. In 2OS Threat Model, the causes to signal abnormality are categorized into three types by the specific parts of the payload, i.e., digital circuit failure(Threat Model A or TMA), analog circuit failure(Threat Model B or TMB) and the hybrid of the previous two(Threat Model C or TMC). These three models can almost cover all the possibilities of signal abnormalities. This type of model is considered as standard signal abnormality model, also known as ICAO Threat Model $^{[6-9]}$. There are three key parameters in the model, i.e., $\Delta$ chip(representing the lead or lag of the spread-spectrum code falling edge relative to the normal position), damped oscillation frequency $f_{d}$ in $\mathrm{MHz}$ (representing the ringing frequency of spread-spectrum code edge) and damper coefficient $\sigma$ in MNerpers/s (representing the attenuation factor of spread-spectrum code edge ringing). The definitions of the above parameters are as shown in Fig .2.

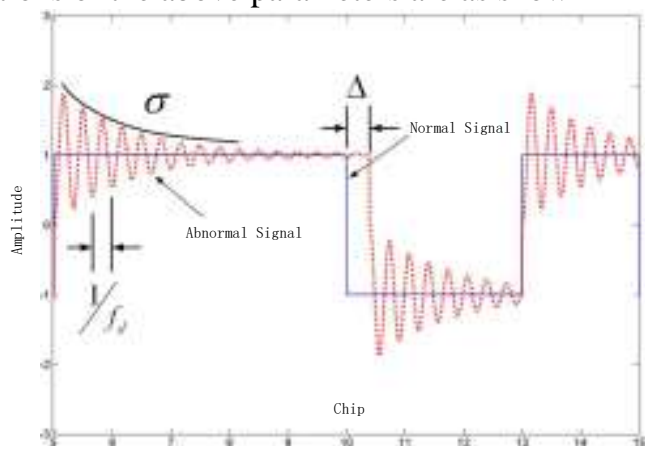

Figure 2. Parameters of ICAO Threat Model

The TMA may be modeled as the sum of the normal sequence and a $\Delta$ sequence, where $\Delta$ sequence is the difference between the normal sequence and the circularshifted normal sequence, which is formulated as

$$
\begin{gathered}
x_{\text {TMA }}(t)=x(t)+x_{\Delta}(t) \\
x_{\Delta}(t)= \begin{cases}\max [x(t-\Delta)-x(t), 0] & \Delta \geq 0 \\
\min [x(t+\Delta)-x(t), 0] & \text { else }\end{cases}
\end{gathered}
$$

The power spectrum of the navigation signal with TMA distortion is as shown in the figure below.

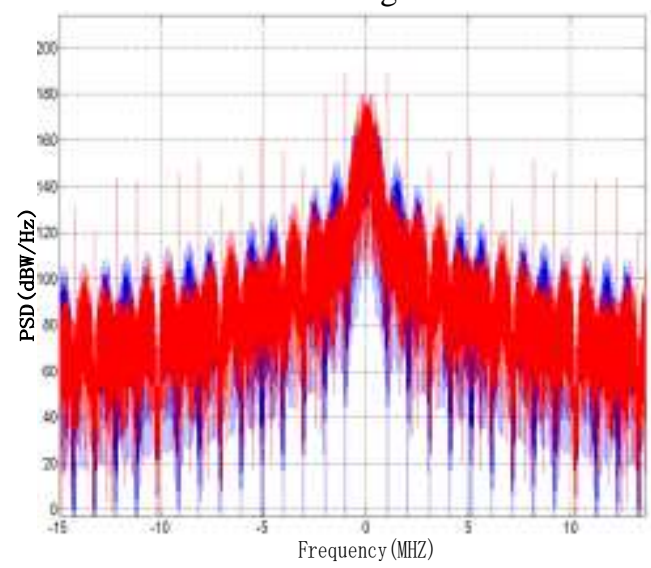

Figure 3. TMA power spectrum density

The TMB may be expressed with a second-order system response as follows:

$$
x_{T M B}(t)=x(t) * h_{\left(\sigma, f_{d}\right)}(t)
$$

Where, $h_{\left(\sigma, f_{d}\right)}(t)$ represents the step response of code edge and may be expressed as follows:

$$
h_{\left(\sigma, f_{d}\right)}(t)=\left\{\begin{array}{lc}
0 & t<0 \\
1-e^{-\sigma t}\left[\cos \left(\omega_{d} t\right)+\sigma / \omega_{d} \sin \left(\omega_{d} t\right)\right] & t \geq 0
\end{array}\right.
$$

Where, $\omega_{d}=2 \pi f_{d}$.

The power spectrum of the navigation signal with TMB distortion is as shown in the figure below.

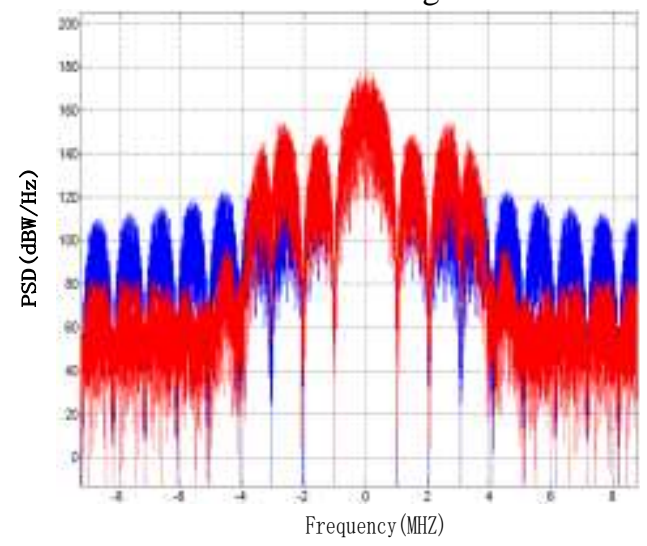

Figure 4. Power spectrum of TMB signal

As the hybrid model of TMA and TMB, TMC may be expressed as

$$
x_{T M C}(t)=\left[x(t)+x_{\Delta}(t)\right] * h_{\left(\sigma, f_{d}\right)}(t)
$$

In general, ICAO Threat Model is mainly established with three parameters, specifically as follows. Threat Model A (TMA) is established with lead/lag parameter $\Delta$. 
It alters the code width of digital signal by changing the rising edge or the falling edge of code to bring about plateau effect to correlation peak. Threat Model B (TMB) is established with resonance frequency $f_{d}$ and attenuation factor $\sigma$. It simulates the failure of analog circuit with second-order oscillation and induces peak distortion or generation of wrong correlation peak value by changing the two parameters. Threat Model C (TMC) is a hybrid model of the above two. It is subject to the changes of $\Delta$, $f_{d}$ and $\sigma$ jointly, as shown in Fig .5.

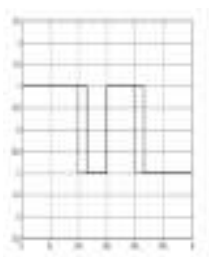

(B)TMA

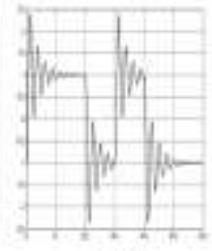

(b) TSE B

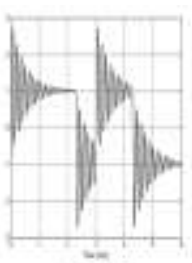

(4) TMC
Figure 5. Three categories of chip waveform distortion

The Annex 6, Vol. 10 of the Convention on International Civil Aviation provides the parameters for ICAO Threat Model, which are so far already approved by the ICAO, as shown in Table 1.

TABLE I. PARAMETER VALUES RANGE OF ICAO THREAT MODEL

\begin{tabular}{|l|c|c|}
\hline \multirow{2}{*}{ Items } & \multicolumn{2}{|c|}{ Parameters } \\
\cline { 2 - 3 } & GPS & GLONASS \\
\hline \multirow{2}{*}{ TMA } & $-0.12 \leq \Delta \leq 0.12$ & $-0.11 \leq \Delta \leq 0.11$ \\
\hline \multirow{2}{*}{ TMB } & $\Delta=0,4 \leq f_{d} \leq 17,0.8 \leq \sigma \leq 8.8$ & $\Delta=0,4 \leq f_{d} \leq 17,0.8 \leq \sigma \leq 8.8$ \\
\hline \multirow{2}{*}{ TMC } & $-0.12 \leq \Delta \leq 0.12$ & $-0.11 \leq \Delta \leq 0.11$ \\
& $7.3 \leq f_{d} \leq 13,0.8 \leq \sigma \leq 8.8$ & $10 \leq f_{d} \leq 20,2 \leq \sigma \leq 8$ \\
\hline
\end{tabular}

\section{TIME-DOMAIN WAVEFORM DISTORTION MODEL OF SATELLITE NAVIGATION SIGNAL}

The time-domain abnormal waveform of navigation signal described in the previous section might happen to any unit of the payload. TMA is generally caused by unequal width of baseband spread-spectrum codes "1" and " 0 " in digital baseband circuit; TMB is generally caused by abnormality in the analog circuit of satellite navigation payload; TMC is a hybrid of the above two. However, it is very difficult to simulate the TMB in an analog circuit in engineering practice. This paper proposes a modeling technique to simulate $\mathrm{TMB}$ in a digital circuit. The hardware implementation structure of time-domain waveform distortion on the baseband FPGA generated by a satellite navigation simulator is shown in Fig .6, where the white box represents the simulation module of normal navigation signal and the gray box represents the simulation module of TMA and TMB.

In TMA, the width difference between baseband spread-spectrum codes " 1 " and " 0 " in a digital baseband circuit is achieved by changing the dynamic clock of the base logic of signal simulator. As for TMB, the secondorder damp oscillation model is essentially equivalent to a second-order $\mathrm{S}$ function ${ }^{[10]}$.

$$
\begin{gathered}
\mathrm{H}(\mathrm{s})=\frac{\left(\omega_{0}\right)^{2}}{s^{2}+2 \zeta \omega_{0} s+\left(\omega_{0}\right)^{2}} \\
\omega^{2}=\sigma^{2}+\left(2 \pi f_{d}\right)^{2}
\end{gathered}
$$

其中 $\sigma=\zeta \omega_{0}, f_{d}=\frac{\omega_{0}}{2 \pi} \sqrt{1-\zeta^{2}}$

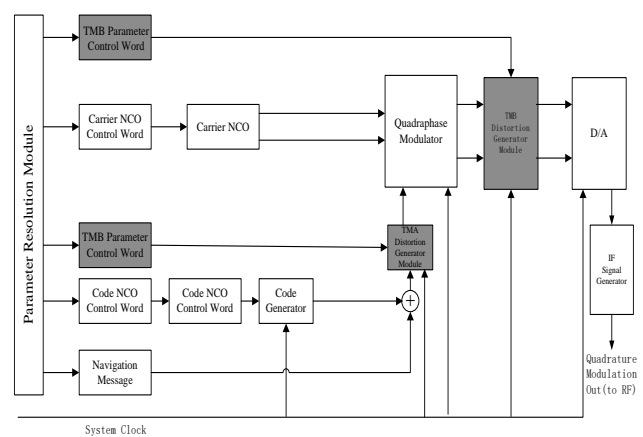

Figure 6. Hardware implementation structure of time-domain waveform distortion

Therefore, it is possible to achieve TMA time-domain waveform distortion by inserting a TMA distortion generation module under the control of system clock between the message modulation module and the quadrature modulation module in Fig .6, as shown in Fig .7. Similarly, it is possible to achieve TMB time-domain waveform distortion by inserting a TMB distortion generation module between the quadrature modulation module and the D/A module in Fig .6. The TMB distortion generation module is equivalent to an IIR filter in nature, as shown in Fig .8.

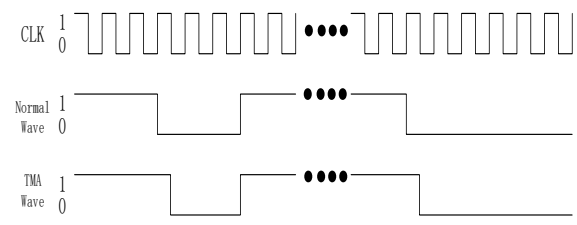

Figure 7. Time sequence of TMA

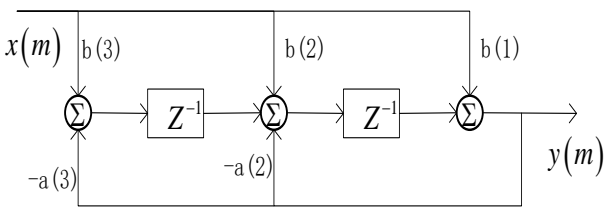

Figure 8. Filter implementation of TMB

\section{4 SimULATION RESUlt OF NAVIGATION SignAL WAVEFORM DISTORTION IN TIME DOMAIN}

TMA abnormality requires the falling edge of spreadspectrum code to be lead or lag by $\Delta$ chip. The waveform of TMA in-circuit emulation $(\Delta=0.12$ and -0.12 respectively) are shown in Fig .9 and Fig .10 respectively. The power spectrums of normal signal and lag TMA signal under 5MHz sweep width are shown in Fig .11. 


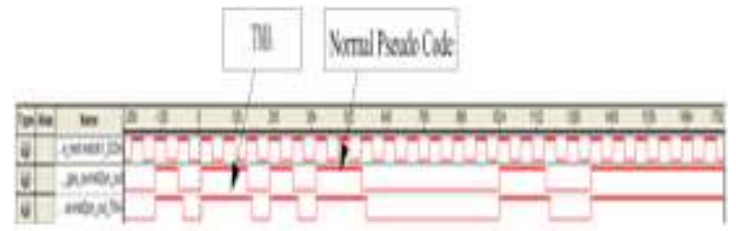

Figure 9. TMA lagged by 0.12 chip

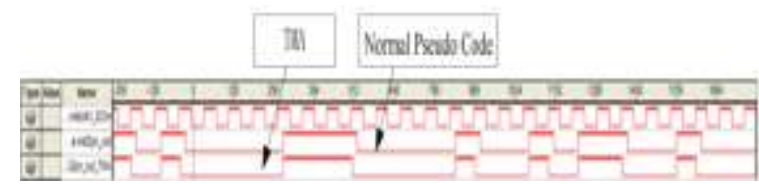

Figure 10. TMA led by 0.12 chip

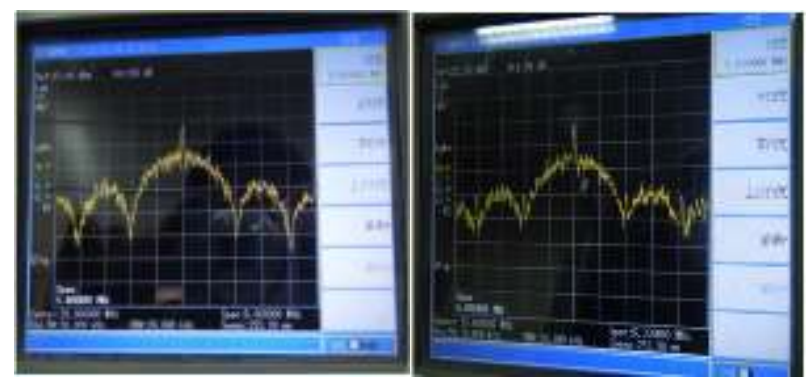

Figure 11. Power spectrums of normal signal and TMA signal lagged by 0.12 chip under $5 \mathrm{MHz}$ sweep width

As shown in Fig .9 and Fig .10, lag or lead happened with the falling edge of the in-circuit emulation waveform of the simulated TMA signal. Observing the burrs between lobes on the spectrum shown in Fig .11, it appears that the spectrum is in agreement with the simulation result shown in Fig .3 in the previous section.

TMB may be expressed with a second-order damped oscillation response having two parameters $\left(f_{d}\right.$ and $\sigma$ ). The waveform of TMB in-circuit emulation under the conditions of parameters $\sigma=5 \mathrm{MHZ}$ and $f_{d}=4 \mathrm{MHz}$ are shown in Fig .12. The power spectrums of normal signal and TMB signal $\left(\sigma=5 \mathrm{MHZ}\right.$ and $\left.f_{d}=4 \mathrm{MHz}\right)$ are shown in Fig .13.

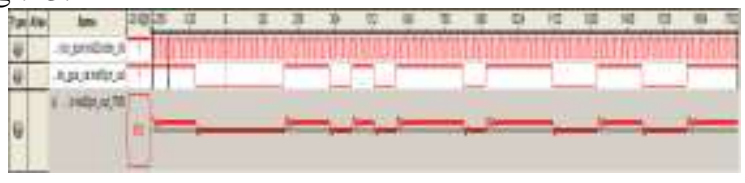

Figure 12. TMB when $\sigma=5 \mathrm{MHZ}$ and $\mathrm{f}_{\mathrm{d}}=4 \mathrm{MHz}$

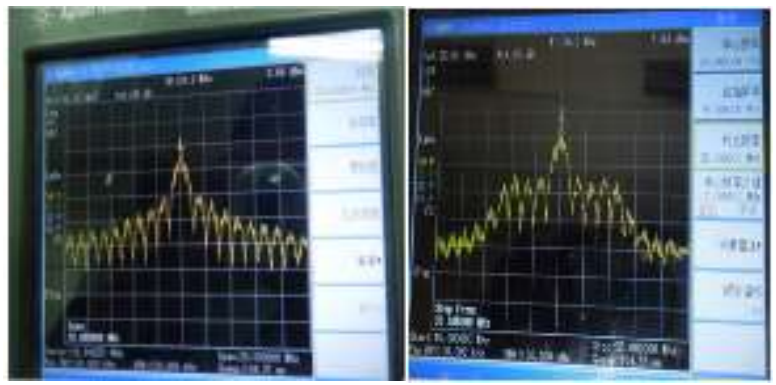

Figure 13. Power spectrums of normal signal and TMB $\operatorname{signal}\left(\sigma=5 \mathrm{MHZ}\right.$ and $\left.\mathrm{f}_{\mathrm{d}}=4 \mathrm{MHz}\right)$

The abnormality implementation results show that the simulated TMB waveform is really a normal signal superposed with a damped oscillation. The true power spectrum shown in Fig .13 is in line with the simulation result shown in Fig .14. Some sidelobes adjacent to the main lobe in the TMB power spectrum diagram are apparently higher than the other sidelobes.

As the hybrid of TMA and TMB, the TMC distortion model generates both digital distortion and analog distortion simultaneously. TMC may be defined with three parameters $\Delta, f_{d}$ and $\sigma$, where $\Delta$ represents the lag of C/A code, $f_{d}$ represents the damped oscillation frequency in a second-order model, which causes randomized jitter of the correlation peak slope, and $\sigma$ represents the damped oscillation coefficient of the second-order model, which defines the jitter strength of the correlation peak slope. The waveform of TMC in-circuit emulation under a number of parameters ( $\sigma=5 \mathrm{MHZ}$ and $\Delta=0.12$ chip) is shown in Fig .14.

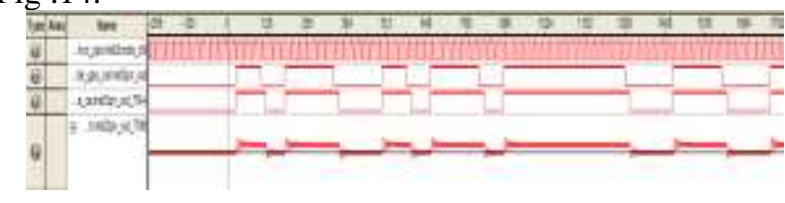

Figure 14. TMB when $\sigma=5 \mathrm{MHZ}, f_{d}=8 \mathrm{MHz}$ and $\Delta=0.12$

The power spectrum diagrams of normal signal and TMC signal $\left(\sigma=5 \mathrm{MHZ}, f_{d}=8 \mathrm{MHz}\right.$ and $\left.\Delta=0.12\right)$ are shown in Fig .15 and Fig .16 respectively. Specifically, the power spectrums of normal signal and TMC signal under $20 \mathrm{MHz}$ sweep width are shown in Fig .15, and those under $5 \mathrm{MHz}$ sweep width are shown in Fig.16.

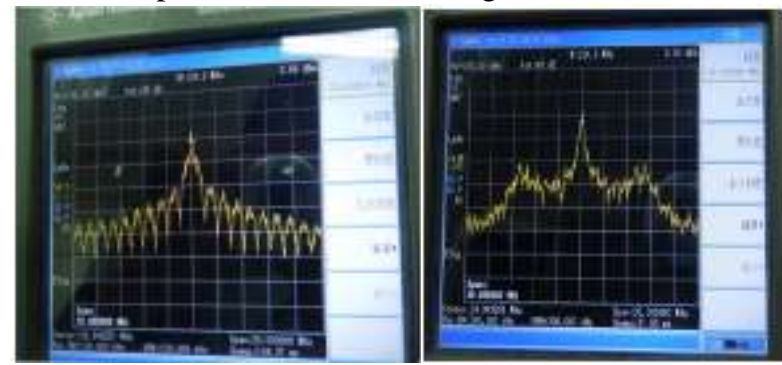

Figure 15. Power spectrums of normal signal and TMC signal unde $20 \mathrm{MHz}$ sweep width

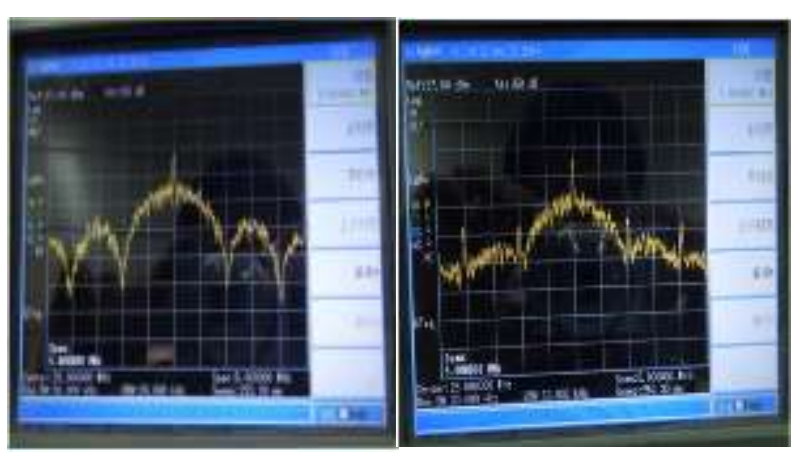

Figure 16. Power spectrums of normal signal and TMC signal under $5 \mathrm{MHz}$ sweep width

As shown in Fig .14, the simulated waveform of TMC has both damped oscillation effect and lag effect of falling edge. Under $20 \mathrm{MHz}$ sweep width, the power spectrum features of TMC hybridized with TMB are visible, i.e., the 
adjacent sidelobes are apparently higher than the other sidelobes. Under $5 \mathrm{MHz}$ sweep width, the power spectrum features of TMC hybridized with TMA are visible, i.e., burrs existing between lobes.

\section{CONCLUSION}

This paper proposes a proposal about modeling timedomain waveform abnormality of satellite navigation signal and how to achieve FPGA implementation. It suggests adding a time-domain waveform distortion module into a signal simulator of true satellite navigation to generate distorted waveform of navigation signal. The simulation results show that the distorted waveform of navigation signal based on FPGA is in agreement with the time-domain waveform and frequency-domain spectrum simulated with MATLAB.

\section{REFERENCES}

[1] C. Edgar,F. Czopek, and B. Barker,"A Co-operative Anomaly Resolution on PRN-19,” ION GPS 2000, Alexandria, VA, 2000, pp2269-2271.

[2] P.Enge, R.E.Phelts, A.Mitelman,"Detecting Anomalous Signals From GPS Satellites,"Working Paper 19, Toulouse, France, 1999.
[3] A.M. Mitelman,"Signal Quality Monitoring for GPS Augmentation Systems,". Stanford University,2004

[4] A.M.Mitelman, R.E.Phelts, D.M.Akos,"LAAS Monitoring for a Most Evil Satellite Failure," ION NTM 1998,Alexandria, VA:,2000,pp 129-134

[5] P.K.Enge, R.E.Phelts, A.M. Mitelman, "Detecting Anomalous signals from GPS Satellites,". ICAO GNSS/P, Toulouse, France 1999.

[6] B. Wang, Y. Pang, H.J. Liu, "Research of Detection Technique of Navigation Signal's Evil Waveform", Journal of Electronics \& Information Technology, val.33, No.7, 2011, pp1713-1717, doi 10.3724/SP.J.1146.2010.01163

[7] G.P. Jin, M.L. Wang, J.J. Fan, "Image of satellite navigation signal quality monitor", Journal of Guilin University of Electronic Technology, vol32(5), 2012,pp358-363, doi:10.3969/j.issn.1673808X.2012.05.004.

[8] J.C. Liu,H.S. Sang,B.B. Xue, "Effect of GNSS Waveform Distortions on SNR", Telecommunication Engineering, vol 1, 2013,doi:10.3969/j.issn.1001-893x.2013.01.006.

[9] Y. XU,J.C. LIU,H.S. SANG, "Receiver Code Tracking Bias Induced by GNSS Waveform Distortions Gnss ", World of China, vol 2, 2014,pp5-8,doi:10.13442/j.gnss.1008-9268.2014.02.002

[10] R.E.Phelts, M. Dennis, "Effects of Signal Deformations on Modernized GNSS Signals,"Journal of Global Positioning Systems,2006, Vol 5,No.12,pp2-10. 